Method Adult patients with a clinical diagnosis of ischaemic cardiomyopathy (ICM), known multi-vessel coronary disease and a left ventricular ejection fraction (LVEF) $\leq 40 \%$ who had undergone both cardiac MRI with late gadolinium enhancement (CMR) and TTE were identified from a local database. TTE and MRI images were analysed by experts blinded to clinical and alternative modality information. End systolic wall thickness (ESWT) and end diastolic wall thickness (EDWT) were measured for each LV segment using the AHA 17-segment model. Viability was adjudicated by the standard late gadolinium enhancement transmurality threshold ( $<50 \%$ viable, 50\% nonviable). Receiver operating characteristic curve analysis was performed to determine the accuracy of ESWT and EDWT in predicting CMR-determined viability. See Figure 1.

Results 237 patient records were screened, 44 met inclusion criteria. Nine of these patients were excluded due to poor image quality (7 TTE, 2 CMR). 35 patients were included for final analysis (72 \pm 8 years, 91\% male). LVEF was $27.8 \% \pm$ 6.4. A total of 595 myocardial segments were available for analysis. 585 (98\%) segments were analysed, 447 (75\%) were assessed as viable and $138(23 \%)$ as non-viable.

Both EDWT and ESWT were significantly greater in viable compared to non-viable segments $(7.6 \pm 2.6 \mathrm{~mm}$ vs. $6.3 \pm$ $2.2 \mathrm{~mm}, \mathrm{p}<0.001$ and $6.9 \pm 2.8 \mathrm{~mm}$ vs. $9.3 \pm 3.2 \mathrm{~mm}$, $\mathrm{p}<0.001$ respectively).

The area under the curve (AUC) for EDWT was 0.646, with an optimal cut point of $6.5 \mathrm{~mm}$. ESWT was more accurate (AUC 0.715, optimal cut point $7.5 \mathrm{~mm}$ ). An ESWT $>10.5 \mathrm{~mm}$ had a specificity of $91 \%$ for viability; only $14 \%$ of segments were above this threshold. See Figure 2.

Conclusion ESWT and EDWT have limited accuracy in predicting segmental viability and the majority of segments would remain within the grey-zone. These data do not support the use of TTE as a filter for proceeding to advanced imaging for viability testing.

Conflict of Interest None

\section{RAPID ACCESS HEART FAILURE CLINIC: IMPACT OF A PHYSIOLOGIST-DELIVERED SERVICE IN A UK DISTRICT GENERAL HOSPITAL} ${ }^{1}$ Hannah Sinclair*, ${ }^{2}$ Melanie Ackrill, ${ }^{2}$ Hiliary Holdsworth, ${ }^{2}$ Chris Chase, ${ }^{2}$ Maxine Guillen,
${ }^{2}$ Louise Bowman, ${ }^{2}$ Louisa Collins, ${ }^{2}$ Chris H Critoph, ${ }^{2}$ Amy CJ Pine. ${ }^{1}$ Royal Bournemouth and
Christchurch Hospital; ${ }^{2}$ Royal Bournemouth Hospital

\subsection{6/heartjnl-2019-BCS.86}

Background National Institute of Clinical Excellence (NICE) Chronic Heart Failure Guideline NG106 recommends NTproBNP measurement in patients with suspected heart failure (HF). NTproBNP 400ng/L mandates referral to a rapid access HF clinic (RAHFC). Target wait is determined byNTproBNP concentration (400-2000ng/L 6 weeks and $>2000 \mathrm{ng} / \mathrm{L}$ within 2 weeks).

In our institution, RAHFC referrals have doubled in a year. There is little published national data regarding adherence to NICE waiting targets. Initial service evaluation found that only $47 \%$ of patients were seen within the target waiting time. However, many patients with lower range NT proBNP $(<1000 \mathrm{ng} / \mathrm{L})$ had no significant cardiac pathology and were discharged. It was proposed this lower range NTproBNP population (400- 1000ng/l) could be safely managed in a cardiac physiologist-delivered RAHFC overseen by a HF specialist. Additonal benefits would be to upskill cardiac physiologists, free consultant time for more severe HF patients and improve waiting times.

Aim To introduce a supervised cardiac physiologist-led HF clinic for patients with a NTproBNP 400ng/L but $<1000 \mathrm{ng} / \mathrm{L}$ and monitor outcomes.

Method An electronic form was developed to guide clinical questioning and data collection. The clinic was initially run by 2 cardiac physiologists and a specialist HF fellow. All clinics were overseen by a consultant HF cardiologist. Demographics, NYHA class, NTproBNP concentration, waiting time, final diagnosis, further investigations and echo results were recorded. Outcomes at 3, 6 and 8 months were retrospectively assessed for safety.

Results 34 patients were seen in the physiologist-delivered HF clinic between Jun'18 and Jan'19. The proportion seen within target waiting time rose from $47 \%$ to $70 \%$. Mean age 81 and $50 \%$ female. Mean NTproBNP was $730 \mathrm{ng} / \mathrm{L}$. 26.5\% $(\mathrm{n}=9)$ were diagnosed with HFPEF and 2.9\% $(n=1)$ HFREF. 32.4\% $(n=11)$ had AF or paroxysmal AF. In $41.2 \% \quad(n=14)$ NTproBNP was felt to be a false positive. Diagnoses included: pulmonary hypertension $(\mathrm{n}=1)$, moderate and severe aortic stenosis $(n=2)$, moderate aortic regurgitation $(n=1)$, bradyarrhythmia requiring pacing $(\mathrm{n}=1)$, hypertrophic cardiomyopathy $(n=2)$, atrial tachycardia $(n=1)$, ectopy $(n=2)$. As cardiac physiologists gained experience, they began to review patients independently and time slots were reduced. No adverse events were recorded.

Conclusion Demand for RAHFC is high and NICE waiting times are often difficult to meet with current service provision. A physiologist-delivered HF clinic proved safe, effective and dramatically reduced waiting times. There is a national shortage of cardiac physiologists, and staff retention and recruitment is difficult. Expanding the role and skill set of our cardiac physiologists has proved popular and good for staff morale.

NTproBNP threshold mandating urgent referral to RAHFC for patients with $\mathrm{AF}$ and those of advanced age may require further research and consideration.

Conflict of Interest None Declared

\section{HETEROZYGOUS GLOBAL DELETION OF PLASMA MEMBRANE CALCIUM ATPASE 1 (PMCA1HT) MAY REDUCE CARDIAC REMODELLING AFTER TRANSVERSE AORTIC CONSTRICTION IN A MURINE MODEL}

${ }^{1}$ Tahir Nazir*, ${ }^{2}$ Florence Baudoin, ${ }^{2}$ Min Zi, ${ }^{2}$ Sukhpal Prehar, ${ }^{2}$ Elizabeth Cartwright. ${ }^{1}$ Division of Cardiovascular Sciences, University of Manchester; ${ }^{2}$ University of Manchester

\subsection{6/heartjnl-2019-BCS.87}

Background Cardiovascular diseases remain a major cause of mortality worldwide. PMCA1 is the most prevalent isoform of PMCA in the heart. Our group has previously generated novel PMCA1 mutant mouse lines using Cre/loxP technology. Cardiomyocyte-specific PMCA1 knockout mice (PMCA1cko) develop severe cardiomyopathy with $\mathrm{HF}$ and heterozygote global PMCA1 knockout mice (PMCA1Ht) have elevated blood pressure. However, whether global heterozygous deletion of PMCA1Ht has any impact on cardiac remodelling after pathological stress resulting from pressure overload 